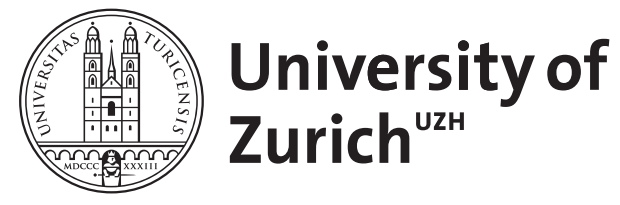
Archive

University of Zurich

University Library

Strickhofstrasse 39

CH-8057 Zurich

www.zora.uzh.ch

Year: 1996

Haemophilus influenzae: a cause of peritonitis in peritoneal dialysis

Neuhaus, T J ; Iselin, H ; Nadal, D

DOI: https://doi.org/10.1093/ndt/11.1.199

Posted at the Zurich Open Repository and Archive, University of Zurich

ZORA URL: https://doi.org/10.5167/uzh-155508

Journal Article

Published Version

Originally published at:

Neuhaus, T J; Iselin, H; Nadal, D (1996). Haemophilus influenzae: a cause of peritonitis in peritoneal dialysis. Nephrology, Dialysis, Transplantation, 11(1):199-200.

DOI: https://doi.org/10.1093/ndt/11.1.199 


\title{
Haemophilus influenzae: a cause of peritonitis in peritoneal dialysis
}

\author{
T. J. Neuhaus ${ }^{1}$, H. Iselin ${ }^{1}$ and D. Nadal ${ }^{2}$ \\ ${ }^{1}$ Division of Nephrology and ${ }^{2}$ Unit of Infectious Diseases, University Children's Hospital, Zurich, Switzerland
}

Key words: child; Haemophilus influenzae; peritoneal dialysis; peritonitis

\section{Introduction}

Peritonitis, a major complication of peritoneal dialysis (PD), is usually caused by coagulase-negative staphylococci, Staphylococcus aureus and streptococci [1,2]. Although infections with Haemophilus influenzae are common in children, peritonitis due to this pathogen is rare [3-5]. We report the first child on PD with a peritonitis due to $H$. influenzae, a fastidious organism requiring adequate culture media for growth.

\section{Case report}

The patient was a 27-month-old child with the Denys-Drash syndrome (triad of Wilms' tumour, diffuse mesangial sclerosis, and male pseudohermaphroditism) [6]. At birth the phenotype was unequivocally female; thus the child was raised as a girl. At the age of 16 months she presented with a right-sided Wilms' tumour stage I and proteinuria. Unilateral nephrectomy was performed, followed by chemotherapy for 4 months. Extratumorous renal tissue showed diffuse mesangial sclerosis. Chromosome analysis revealed a male karyotype $46, X Y$ and a constitutional mutation in the WT1 locus. Ultrasound and computing tomography showed an infantile uterus; however, the gonads could not be classified, even at laparotomy.

End-stage renal failure occurred at the age of 21 months. The second kidney was removed, and automated continuous cycling peritoneal dialysis (CCPD) during the night (PAC-Xtra cycler, Baxter) was commenced. After 1 month the child developed a tunnel infection, without concomitant peritonitis, due to Pseudomonas aeruginosa, which was successfully treated with a 10-day course of intraperitoneal ceftazidime.

At the age of 24 months the child presented afebrile with cloudy dialysate and slight abdominal pain. There

Correspondence and offprint requests to: T. J. Neuhaus, Division of Nephrology, University Children's Hospital, Steinwiesstr. 75, CH-8032 Zurich, Switzerland. was neither a tunnel nor an exit-site infection. Microscopy of the dialysate showed $6700 \times 10^{6} / 1$ white cells. Gram staining was negative. After taking samples of dialysate and blood, intraperitoneal gentamicin and cefazolin were administered. Within $48 \mathrm{~h}$, the dialysate became clear and the white cell count normalized $\left(5-15 \times 10^{6} / 1\right)$.

For bacterial cultures, sediment from $50 \mathrm{ml}$ highspeed centrifuged dialysate was plated onto sheep blood and MacConkey agar plates. In addition uncentrifuged dialysate was instilled into four haemoculture bottles ( $10 \mathrm{ml}$ each; Bactec Plus, Becton Dickinson, Basel, Switzerland), which were immediately enriched with hemin ( $X$ factor) and nicotinamide adenine dinucleotide (NAD, V factor) (Bactec ${ }^{\text {FOS }}{ }^{\mathrm{TM}}$, Becton Dickinson). Dialysate cultures in these bottles grew non-groupable $H$. influenzae biotype 2 , sensitive to both administered antimicrobials. In contrast, the agar plates remained sterile. Haemocultures and a nasal swab (also cultured on chocolate agar) showed no growth. Gentamicin was discontinued and the patient was treated for 10 days with cefazolin, and has been well since.

\section{Discussion}

Haemophilus influenzae is an important pathogen in children. Peritonitis due to this organism has rarely been found in previously healthy $[3,4]$ and in nephrotic children [5]. PD peritonitis due to $H$. influenzae has not been described in children, in contrast to occasional reports from adults [7-10]. The source of infection in our patient is unclear. Tunnel, exit-site, and surrounding skin of the Tenckhoff catheter were unremarkable and there were no signs of upper respiratory tract infection. Peritonitis rate in children on PD is higher than in adults [2]. Since 1979 we have treated 27 children with end-stage renal failure with ambulatory PD. The rate of peritonitis has declined with improving techniques to one episode per 20 months since 1989.

Cultures of peritoneal fluids from patients with PD peritonitis (defined as $>100 \times 10^{6} / 1$ white cells) have been reported as negative in up to $50 \%$ of all episodes $[1,2]$. Relevant reasons include low concentrations of micro-organisms, intracellular survival of and surface tension between bacteria, and presence of antibiotics [1]. Another reason may be the lack of specific growth 
factors in the culture media. Haemophilus spp. are nutritionally fastidious and require either exogenous hemin ( $\mathrm{X}$ factor) or NAD ( $\mathrm{V}$ factor) or both. Chocolate agar but not conventional sheep blood agar contains $\mathrm{X}$ and $\mathrm{V}$ factor in sufficient amounts. Whereas both factors are provided in haemocultures from the patient's blood, they need to be added to the media when culturing dialysate. Thus the use of chocolate agar should routinely be introduced for cultures of dialysate. Direct instillation of (even uncentrifuged) dialysate into haemoculture bottles containing $X$ and $\mathrm{V}$ factor-enriched medium, as done in the present case, may optimize detection of Haemophilus spp.

We conclude that (1) Haemophilus spp. peritonitis does occur in children, and (2) may be missed if bacterial cultures of dialysate are not done with adequate media for the growth of these pathogens.

\section{References}

1. v Graevenitz A, Amsterdam D. Microbiological aspects of peritonitis associated with continuous ambulatory peritoneal dialysis. Clin Microbiol Rev 1992; 5: 35-48
2. Avner ED, Chavers B, Sullivan EK, Tejani A. Renal transplantation and chronic dialysis in children and adolescents: the 1993 Annual Report of the North American Pediatric Renal Transplant Cooperative Study. Pediatr Nephrol 1995; 9: $61-73$

3. Chang MJ, Controni G. Primary pentonitis due to Haemophilus infuenzae type b in a previously healthy child. $J$ Clin Microbiol 1983; 18: 725-726

4. Gorski J, Ingall D, Yogev R. Primary peritonitis associated with Hemophilus influenzae bacteremia in a normal child. Clin Pediatr 1983; 22: 183

5. Rubin HM, Blau EB, Michaels RH. Hemophilus and pneumoccocal peritonitis in children with the nephrotic syndrome. Pediatrics 1975; 56: 598-601

6. Coppes MJ, Huff V, Pelletier J. Denys-Drash syndrome: relating a clinical disorder to genetic alterations in the tumor suppressor gene WT1. J Pediatr 1993; 123: 673-678

7. Gokal R, Francis DMA, Goodship THJ et al. Peritonitis in continuous ambulatory peritoneal dialysis. Lancet 1982; 2: 1388-1391

8. Spencer RC. Infections in CAPD. J Med Microbiol 1988; 27 : $1-9$

9. Ferrari R, Dasgupta MK. A case of CAPD peritonitis due to Hemophilus influenzae. Perit Dial Int 1993; 13: 323-324

10. Maxwell PH, Abbott J, Koffman CG, Dave J. Haemophilus influenzae as a rare cause of CAPD peritonitis. J Infect 1993; 26: $340-34$

Received for publication: 29.895

Accepted: 6.9.95 